\title{
The anticipation of pain at a specific location of the body prioritizes tactile stimuli at that location.
}

\author{
Authors: Charlotte Vanden Bulcke*, Stefaan Van Damme*, Wouter Durnez*, Geert \\ Crombez* \\ Department: *Department of Experimental-Clinical and Health Psychology, Ghent \\ University
}

Text pages:20

Figures/tables:3

\section{Corresponding author:}

Charlotte Vanden Bulcke, Ghent University, Faculty of Psychology and Educational

Sciences, Department of Experimental-Clinical and Health Psychology, Henri Dunantlaan 2, 9000 Ghent, Belgium.

E-mail: Charlotte.VandenBulcke@UGent.be

Phone number: +32(0)9-264 86 11. Fax: +32(0)9-264 6489 . 


\section{Introduction}

Attention is a central component in pain theories aiming to explain amplified pain perception, disability, and distress $[4,10,14,21,32]$. Influential is the idea that patients with chronic pain are characterized by hypervigilance, referring to a preoccupation with bodily threat signals as a result of which attention prioritizes pain-related information at the cost of other environmental demands [8,35]. A recent meta-analysis [9] of studies measuring attentional prioritization of pain-related information indicated that the available evidence supporting this idea is weak. However, the paradigms typically used in these studies may not be suitable to activate pain schemata/memories, as they only assess the prioritization of painrelated words or pictures, and not of pain or somatosensory stimuli. Hence, the use of somatosensory attention paradigms has been recommended $[9,32]$. The present study is a step into this endeavor.

If fearful anticipation of pain leads to heightened attention to pain-related information $[8,14,32]$, we hypothesized that this would result in the prioritization of -even innocuous somatosensory input at body locations where pain is expected to occur. Indeed, according to Titchener's [26] law of prior entry, stating that attended stimuli come to consciousness more quickly than unattended stimuli (see [23]), we may expect that one becomes more quickly aware of somatosensory stimuli in a particular location of the body where pain is expected, relative to somatosensory stimuli in other regions of the body. Evidence for our hypothesis is yet limited. In a study of Crombez and colleagues (1998), healthy volunteers were led to believe that a very intense, almost intolerable painful stimulus could occur at one particular location of the body. As a result, a mildly painful stimulus at that particular location interfered more with the performance of an ongoing, cognitive task, than pain stimuli at another location [6]. However, no studies have investigated whether the anticipation of pain makes one more 
quickly aware of non-painful somatosensory information in the threatened body part relative to other body parts.

The aim of the present study was to specifically test this idea. We investigated in healthy persons whether the anticipation of (experimentally induced) pain in one hand results in a prioritization of innocuous tactile stimuli at that hand, using a tactile Temporal Order Judgment (TOJ) task [24]. Participants were required to report which one of two tactile stimuli, one administered to each hand at a range of different stimulus onset asynchronies (SOAs), was perceived first. Performance on this task provides information about which hand is prioritized by attention (see $[23,30]$ ). Participants were instructed that the color of a cue (one of two colors) signaled the possible occurrence of pain on one hand (threat trials). The other color of the cue signaled that no pain would follow (control trials). We hypothesized that in threat trials tactile stimuli would be perceived earlier in time on the hand where pain was expected than on the "neutral" hand.

\section{Method}

\section{Participants}

Twenty undergraduate psychology students (19 female and 1 male; mean age, 18.3 years; all white Caucasian) participated to fulfill course requirements. All participants had normal or corrected-to-normal vision and normal hearing. All but 2 were right-handed as reported by self-report. Sixteen participants reported to have experienced pain during the last six months (average of 12 days in 6 months). Seven participants reported to feel pain at the moment of testing, but the average rating of the intensity of the pain for these 7 participants was low $(\mathrm{M}=2.29, \mathrm{SD}=1.38)$ on a Likert scale where 0 indicated 'no pain' and 10 'worst pain ever'. Participants rated their general health on average as 'very good' and none of all participants reported to have a current medical or mental disorder. All participants gave informed consent 
and were informed to be free to terminate the experiment at any time. The study protocol was approved by the Ethics Committee of the Faculty of Psychology and Educational Sciences of Ghent University. The experiment lasted for approximately 1 hour and 15 minutes.

\section{Apparatus and stimulus material}

Tactile stimuli (10 ms duration; 200Hz) were presented by means of two resonant-type tactors (C-2 TACTOR, Engineering Acoustics, Inc., Florida, http://www.eaiinfo.com/ ) consisting of a housing of $3.05 \mathrm{~cm}$ diameter and $0.79 \mathrm{~cm}$ high, with a skin contactor of 0.76 $\mathrm{cm}$ diameter. Prior to the start of the experiment, the perceived stimulus intensities at both tactor locations were individually matched [36]. This was done by means of a double random staircase procedure, based on the 'simple up-down method' of Levitt [15]. In a first phase, 24 stimuli presented on the left hand were judged relative to a reference stimulus with maximum intensity (power $=0.21 \mathrm{Watt}$ ) on a 5-point Likert scale ranging from 1 ('no sensation') to 5 ('maximum intensity'). The intensity that elicited an averaged rating of 3 was used as the stimulus intensity for the left hand, and was the reference stimulus for the second phase. In the second phase 24 stimuli on the right hand were judged relative to the reference stimulus on the left hand on a 5-point Likert scale ( $1=$ 'more than less strong', $2=$ 'less strong', $3=$ 'equally strong', $4=$ 'stronger', 5= 'much stronger'). The intensity that elicited an averaged rating of 3 was used as the intensity of the stimulus at the right hand.

Painful stimuli were electrocutaneous stimuli delivered by constant current stimulators (Digitimer DS5 2000, Digitimer Ltd, England, http://www.digitimer.com/index.htm). Electrocutaneous stimuli consisted of trains of $20 \mathrm{~ms}$ sinusoid pulses with a frequency of 50 $\mathrm{Hz}$, and were delivered via two lubricated Fukuda standard $\mathrm{Ag} / \mathrm{AgCl}$ electrodes $(1 \mathrm{~cm}$ diameter $)$ for $200 \mathrm{~ms}$. Intensity of the electrocutaneous stimuli was determined for each participant individually by means of a random staircase procedure. For each hand, 20 electrocutaneous stimuli were presented to participants (start intensity between 0 and $1.5 \mathrm{~mA}$ ) and self-reports 
were collected on an 11 -point Likert scale ( $0=$ 'no sensation'; $10=$ 'unbearable pain'). The pain intensity that elicited an average rating of 7 was selected as the pain stimulus for the proper experiment [1].

\section{Tactile Temporal Order Judgment paradigm}

The task was programmed and controlled by the INQUISIT Millisecond software package (Inquisit 3.0, Millisecond Software LLC, Seattle, WA, http://www.millisecond.com//) on a laptop (HP Compaq nc 6120). Each trial began with a fixation cross (1000 ms) in the middle of the screen, followed by a colored cue (1000 ms), indicating whether or not a painful electrocutaneous stimulus could follow on one hand. A yellow rectangle $(10$ by $10 \mathrm{~cm})$ indicated that no electrocutaneous stimulus would follow (control trials). A blue rectangle (10 by $10 \mathrm{~cm}$ ) indicated that a painful electrocutaneous stimulus on one hand could follow (threat trials). In $10 \%$ of all threat trials, the pain stimulus was actually delivered instead of the two tactile stimuli. Participants were not informed about the proportion of pain stimuli. On trials without pain stimulus (90\% of threat trials and all control trials), two tactile stimuli were administered, one on each hand. These stimuli were separated in time by one of 10 randomly assigned stimulus onset asynchronies (SOAs) ranging from -120 to $+120 \mathrm{~ms}(-120,-60,-30,-$ $15,-5,+5,+15,+30,+60,+120 \mathrm{~ms}$; negative values indicate that the left hand was stimulated first) [see also 16,29]. Participants were asked to report aloud on which hand the tactile stimulus was presented first. When a pain stimulus replaced a tactile TOJ trial, participants were informed that no response had to be given. Responses were coded by the experimenter using a keyboard.

\section{Procedure}

Participants were tested individually. First, the TOJ task was explained to the participants. They were also informed that an electrocutaneous stimulus would be used during the experiment and that "most people find this kind of stimulation unpleasant". After participants 
gave their informed consent, they were seated in front of the experimental apparatus. The forearms were positioned symmetrically on the table. The tactors were placed on the metacarpal of each hand. Electrodes were attached to both hands between thumb and index finger, in the sensory territory of the superficial radial nerve. The skin at the electrode sites was first abraded with a peeling cream (Nihon Kohden, Tokyo, Japan) to reduce skin resistance. Participants were instructed that the color of a cue (one of two colors) signaled the possible occurrence of pain on one hand. The other color of the cue signaled that no pain would follow. Before the start of each block, participants were informed on which hand (left or right) they could expect painful stimuli. Participants had to report aloud which one of two tactile stimuli, one administered to each hand was presented first. Accuracy of participants' responses was emphasized, rather than speed. Participants wore headphones (Wesc, Conga) during the experiment. White noise $(42.2 \mathrm{~dB}$ ) was presented continuously through the headphones to mask the noise resulting from the operation of the tactors. The participants were not given any feedback about their performance.

The session began with a practice block of twenty-three trials (1 trial per SOA for control trials; 1 trial per SOA for threat trials; 3 electrocutaneous trials). Following this, four blocks of 105 trials ( 5 trials per SOA for control trials; 5 trials per SOA for threat trials, 5 pain trials) were randomly presented with the two possible locations of pain (left hand or right hand) alternating between blocks and counterbalanced between participants.

\section{Self-report measures}

After each test phase, participants had to rate several questions about concentration ('To what extent have you made an effort to this task?', 'To what extent did you concentrate on this task?'), attention to painful/tactile stimuli ('To what extent did you pay attention to the painful/tactile stimuli?'), pain experience ('How painful did you find the electrocutaneous stimuli?'), anxiety ('How anxious were you during this block?'), fatigue ('To what extent did 
you find this task tiring?') on an eleven-point numerical rating scale (anchored $0=$ not at all and $10=$ very strongly). As a manipulation check, we were especially interested in the ratings of fear ('To what extent were you afraid that the blue/yellow cue would be followed by a painful stimulus?') and expectations ('To what extent did you expect that the blue/yellow cue would be followed by a painful stimulus?'). Participants were also asked to complete the Pain Vigilance and Awareness Scale (PVAQ) [16,20] and the Pain Catastrophizing Scale (PCS) $[7,25]$. These data were collected for meta-analytical purposes and are not reported in detail here.

\section{Results}

\section{Self-report data and manipulation check}

Participants rated the electrocutaneous stimuli as moderately painful $(\mathrm{M}=5.38, \mathrm{SD}=$ 1.77). Furthermore, they reported to be more afraid during threat trials $(M=5.86, S D=1.76)$ than during control trials $(\mathrm{M}=0.05, \mathrm{SD}=0.17)\left(t_{19}=14.85, p<0.01 ; d=3.32\right.$ [95\% CI: $2.20,4.44])$. Finally, they expected a painful electrocutaneous stimulus more strongly during threat trials $(\mathrm{M}=6.16, \mathrm{SD}=1.69)$ than during control trials $(\mathrm{M}=0.11, \mathrm{SD}=0.25)\left(t_{19}=\right.$ $15.31, p<0.01 ; d=3.43$ [95\% CI: 2.28, 4.58]). Mean questionnaire scores were $10.90(\mathrm{SD}=$ 11.16) for the PCS and $36.30(\mathrm{SD}=8.96)$ for the PVAQ.

\section{TOJ data handling}

In a TOJ task, it is recommended [22,24] to exclude participants from statistical analysis when any of the PSS values is greater than the highest SOA $( \pm 120 \mathrm{~ms})$ and when participants have less than $80 \%$ accuracy on the trials with the largest SOA tested ( $\pm 120 \mathrm{~ms})$. No participants had to be excluded for these reasons. Trials following trials with electrocutaneous stimulation were removed from data analysis to avoid that (1) potential effects would be 
mainly driven by trials directly following painful stimulation or (2) after-effects of pain would interfere with the tactile TOJ (max. $10 \%$ of all trials).

The analyses were based on the procedure described by Spence and colleagues [24] (see also [22,30]). The proportions of 'right-hand-first' responses for all trials at each SOA, for threat presented on the right hand, and the proportion of 'left-hand-first' responses for all trials at each SOA, for threat presented on the left hand, were converted into the corresponding z-scores using a standardized normal distribution. The best-fitting straight line was computed for each participant and the derived slope and intercept values were used to compute the point of subjective simultaneity (PSS) and the just noticeable difference (JND) values for the subsequent statistical analyses (see Fig. 1). The PSS refers to the point at which observers report the two events (right hand first and left hand first) equally often. This is considered equivalent to the (virtual) SOA at which participants perceive the two stimuli as occurring at the same time. We recoded the PSS data so that a positive value indicates that the stimulus contralateral to the side of threat had to be presented first in order for both stimuli to be perceived as simultaneous. As a result, a positive PSS means that stimuli on the threatened hand are perceived more rapidly than stimuli on the other hand. The JND is monotonically related to the slope of the psychometric function and indicates the interval needed to achieve $75 \%$ correct performance, and as such provides a standardized measure of the sensitivity of participants' temporal perception. A repeated measures analysis of variance (ANOVA) with the factor Trial type (threat versus control) was performed on the PSS and JND data (note that we had no specific hypotheses concerning the JND index). For ease of comparison with the norms of Cohen [5], we calculated effect sizes for dependent samples using the formula of Dunlap and colleagues [3]. We determined whether Cohen's $d$ was small (0.20), medium (0.50), or large (0.80) [5]. We also report the $95 \%$ confidence intervals $(95 \% \mathrm{CI})$ of the effect sizes. 


\section{Insert Figure 1}

\section{PSS deta}

The main effect of Trial Type was significant $(F(1,19)=9.71, p<0.01)$, with threat trials showing a larger PSS $(M=25.37 \mathrm{~ms}, \mathrm{SD}=20.48)$ than control trials $(\mathrm{M}=8.71 \mathrm{~ms}, \mathrm{SD}=$ 11.15) $(d=0.70$ [95\% CI: $0.21,1.19])$ (see Fig. 2). These results suggest that tactile stimuli on the "pain" hand were prioritized. Table 1 represents the PSS values for threat and control trials for each participant individually. The PSS from both control and threat trials differed significantly from the actual point of simultaneity $(0 \mathrm{~ms})$, respectively $t(19)=3.49, p<0.01$ and $t(19)=5.54, p<0.001$. These results suggest that even when participants were cued that no painful stimulus would follow (control trials), they perceived tactile stimuli on the "pain" hand faster than on the "neutral" hand. When excluding the two left-handed participants or the only male participant, the results remain the same. Also when the data are analyzed without exclusion of trials immediately following a pain stimulus, the results remain the same. Finally, no significant associations were found between the PSS values and the scores on the PVAQ and PCS.

Insert Table 1 and Figure 2

\section{$J N D$ data}

There was a main effect of Trial type $(F(1,19)=6.90, p<0.05)$, revealing that the JND was larger in threat trials $(\mathrm{M}=51.35, \mathrm{SD}=24.85)$ compared to control trials $(\mathrm{M}=39.93, \mathrm{SD}$ $=18.81)(d=0.59$ [95\% CI: 0.11, 1.06]) (see Fig. 2). When excluding the two left-handed participants or the only male participant, the results remain the same. Also when the data are 
analyzed without exclusion of trials immediately following a pain stimulus, the results remain the same. Finally, no significant associations were found between the JND values and the scores on the PVAQ and PCS.

\section{Discussion}

The present study investigated whether one becomes more quickly aware of innocuous somatosensory stimuli in a region of the body where pain is anticipated. Our data indicate that when participants made judgments regarding which of two tactile stimuli had been presented first, stimuli on the "neutral" hand had to precede stimuli on the hand on which pain was expected for the two stimuli to be perceived as simultaneous (PSS). This indicates that people perceive stimuli presented on the hand on which pain was expected more rapidly than stimuli presented on the "neutral" hand. Crucially, this effect was significantly larger in threat trials than in control trials. The effect was medium to large according to conventional norms for effect sizes. Thus, when participants anticipated pain at a particular location of the body, they became more quickly aware of innocuous somatosensory signals at that location of the body. To the best of our knowledge, it is the first study demonstrating that anticipating pain in a particular body part prioritizes somatosensory input at that body part. According to the prior entry hypothesis [26], attended stimuli are perceived more rapidly than simultaneously presented stimuli that are not attended. Our results thus indicate that tactile attention was prioritized towards the location of the body where pain was expected.

The current findings fit well in a recently developed neurocognitive model of attention to pain [14]. The model incorporates two modes on how attention is prioritized by pain-related information. On the one hand, bottom-up capture of attention by pain is an involuntary process that demands attention, interrupts ongoing goals, and prioritizes appropriate behaviors 
to escape from bodily threat. Top-down attention, on the other hand, is an intentional and goal-directed process that prioritizes information relevant for current goals or actions. It is proposed that top-down selection occurs by means of an attentional set, defined as a mental set of stimulus features that participants use to identify goal-relevant stimuli. All stimuli that meet one or more of these features will capture attention. In the present study, the anticipation of pain at a particular body location may have led to increased somatosensory expectations within participants' attentional set, as a result of which they prioritized somatosensory input at that location.

The paradigm proposed in this study may be useful to assess hypervigilance in chronic pain patients. It is typically assumed that chronic pain patients are characterized by an excessive focus of attention for - even innocuous - bodily sensations, although convincing evidence is currently lacking $[18,19]$. In line with the neurocognitive model of attention to pain [14], it may be that chronic pain patients maintain features of excessive somatosensory expectations within their attentional set for particular locations of the body where they expect to feel pain. For example, patients with chronic lower back pain (CLBP) or temporomandibular joint dysfunction (TMD) may tend to focus their attention to bodily sensations specifically in the back or face, respectively. When applying the TOJ paradigm in these samples, pairs of tactile stimuli could consist of a stimulus at a pain-relevant location (e.g., back in CLBP, jaw in TMD) and a stimulus at a pain-irrelevant location. Hypervigilance should then be reflected by a bias of attention towards the pain-relevant location, i.e., prioritization of tactile stimuli in the pain-relevant compared to the pain-irrelevant region of the body.

Our study marks a shift in research methods to investigate attentional mechanisms related to pain in two ways. First, most of the previous work in this area has focused on visual attention, i.e. the measurement of biases in attention to pain-related visual stimuli such as 
words, pictures, or conditioned cues. A meta-analysis of Crombez and colleagues [9] about attentional bias to pain-related information indicated that chronic pain patients display an attentional bias towards pain-related words or pictures, but this bias was of a small effect size, and did not significantly differ from that of control groups. Visual stimuli may, however, not be suitable to activate pain schemata/memories, and therefore, research using somatosensory attention paradigms is recommended $[7,9,32]$. Our study is one of the first doing so. Second, behavioral studies investigating hypervigilance typically rely on reaction times. Such an approach may be less suitable for clinical populations. It is well-known that chronic pain patients are often characterized by cognitive impairment and psychomotor slowing, which increases reaction time variability and reduces sensitivity to detect effects [29]. Here, a tactile TOJ task was used, which provides a sensitive measure for detecting biases in spatial attention irrespective of response speed [23,24,30]. Such approach may prove more useful for further research in clinical samples.

A number of issues deserve further discussion. First, this study was conducted with healthy volunteers, using experimental pain. Therefore, one must be cautious in generalizing the results to chronic pain patients. Our findings need extension in clinical pain populations. Second, in this study we specifically examined the effects of anticipated pain on tactile attention. As it has been shown that tactile perception may be reduced by actual pain, either experimental [2,12] or chronic [17], an intriguing question is how the presence of pain during tactile TOJ's would affect attentional prioritization effects. Third, analysis of the JND data revealed that participants were less accurate in making tactile TOJs on trials in which bodily threat was induced compared to control trials. Although we had no specific hypotheses regarding the JND, this reduced accuracy in tactile TOJs following the anticipation of pain is in line with studies showing that painful somatosensory stimuli interfere with task performance (e.g., $[6,27,28,34])$. Forth, it should be noted that also in control trials the PSS 
differed significantly from the actual point of simultaneity (0ms). In other words, even when participants were cued that no painful stimulus would follow, they perceived tactile stimuli on the "pain" hand faster than on the "neutral" hand, suggesting that also in these trials attention was prioritized - to some extent - to the "pain" hand. A possible explanation could be that participants in a so-called 'safe situation' still fear that a painful stimulus would follow. Although the self-report measures do not seem to confirm this (participants almost never expected a painful electrocutaneous stimulus during control trials), the retrospective nature of these ratings may have prevented the detection of subtle expectations during the control trials. Fifth, we did not use a control condition in which a non-painful somatosensory stimulus at a specific location of the body was anticipated. Although it has already been demonstrated that visual cues signaling a painful stimulus attract more attention than visual cues signaling a non-painful tactile stimulus [28,31], it is possible that part of the prioritization effect in our study is not unique to the anticipation of pain. It is recommended that future studies should include an adequate control condition. Sixth, despite the fact that the statistical analysis confirmed our hypothesis, with a moderate to large effect size, we noticed substantial individual variability. It would be interesting to examine which variables may explain this variability. We recommend follow-up studies in which potential theoretically relevant moderators, such as the affective-motivational relevance of pain [8,32], are experimentally manipulated. Finally, our study does not allow conclusions about how close somatosensory stimuli should be to the pain location in order to be prioritized by attention. Therefore, it would be interesting to investigate in future studies if the prioritization of somatosensory attention is limited to the exact location of nociception, or if it is generalized to the whole body part or even the whole side of the body.

In conclusion, the current findings indicate that the anticipation of pain at a particular location of the body results in prioritization in time of innocuous somatosensory sensations at 
that particular location of the body. This suggests that our brain prioritizes tactile information at threatened body parts. The paradigm used here may be a promising tool to investigate somatosensory hypervigilance in clinical populations.

\section{Acknowledgements}

This study was supported by a Research Grant (3G005611) by the Research Foundation Flanders (FWO) awarded to S. Van Damme and G. Crombez. There are no conflicts of interest that may arise as a result of this research. 


\section{References}

[1] Arntz A, Dreessen L, De Jong P. The influence of anxiety on pain: attentional and attributional mediators. Pain 1994;56:307-314.

[2] Bolanowski SJ, Gescheider GA, Fontana AM, Niemic, JL, Tromblay JL. The effects of heat-induced pain on the detectability, discriminability, and sensation magnitude of vibrotactile stimuli. Somatosens Mot Res 2001;18:5-9.

[3] Borenstein M, Hedges LV, Higgins JPT, Rothstein HR. Introduction to Meta-analysis. Wiley, West Sussex, 2009.

[4] Chapman CR. Pain: the perception of noxious events. In: Sternbach RA, editor. The Psychology of Pain. New York, 1978. pp. 169-202.

[5] Cohen J. Statistical power analysis for the behavioral sciences. McGraw-Hill, San Diego, CA, 1988.

[6] Crombez G, Eccleston C, Baeyens F, Eelen P. When somatic information threatens, catastrophic thinking about pain enhances attentional interference. Pain 1998; 75: 187-198.

[7] Crombez G, Hermans D, Adriaensen H. The emotional strooptask and chronic pain: what is threatening for chronic pain sufferers. Eur J Pain 2000;4:37-44.

[8] Crombez G, Van Damme S, Eccleston C. Hypervigilance to pain: an experimental and clinical analysis. Pain 2005;116:4-7.

[9] Crombez G, Van Ryckeghem DML, Eccleston C, Van Damme S. Attentional bias to painrelated information: a meta-analysis. Pain 2013;154:497-510.

[10] Eccleston C, Crombez G. Pain demands attention: A cognitive-affective model of the interruptive function of pain. Psych Bull 1999;125:356-366.

[11] Eccleston C, Grombez G, Aldrich S, Stannard C. Attention and somatic awareness in chronic pain. Pain 1997;72:209-215. 
[12] Harper D, Hollins M. Is touch gating due to sensory or cognitive interference? Pain 2012; 153: 1082-1090.

[13] Hollins M, Harper D, Gallagher S, Owings EW, Feng Lim P, Miller V, Siddiqi MQ, Maixner W. Perceived intensity and unpleasantness of cutaneous and auditory stimuli: An evaluation of the generalized hypervigilance hypothesis. Pain 2009;141: 215-221. [14] Legrain V, Van Damme S, Eccleston C, Davis KD, Seminowicz DA, Crombez G. A neurocognitive model of attention to pain: behavioral and neuroimaging evidence. Pain 2009; 144:230-232.

[15] Levitt H. Transformed up-down methods in psychoacoustics. J Acoust Soc Am 1971;49:467-477.

[16] McCracken, LM. Attention to pain in persons with chronic pain: A behavioural approach. Behav Ther 1997;28:271-284.

[17] Moseley GL. I can't find it! Distorted body image and tactile dysfunction in patients with chronic back pain. Pain 2008;140:239-243.

[18] Peters ML, Vlaeyen JWS, van Drunen C. Do fibromyalgia patients display hypervigilance for innocuous somatosensory stimuli? Application of a body scanning reaction time paradigm. Pain 2000;86:283-292.

[19] Peters ML, Vlaeyen JWS, Kunnen AMW. Is pain-related fear a predictor of somatosensory hypervigilance in chronic low back pain patients? Behav Res Ther 2002;7:2330.

[20] Roelofs J, Peters ML, Muris P, Vlaeyen JWS. Dutch version of the Pain Vigilance and Awareness Questionnaire: validity and reliability in a pain-free population. Behav Res Ther 2002;40:1081-1090.

[21] Rollman GB. Perspectives on hypervigilance. Pain 2009;141:183-184. 
[22] Shore DI, Gray K, Spry E, Spence C. Spatial modulation of tactile temporal-order judgments. Percept 2005;34:1251-1262.

[23] Spence C, Parise C. Prior-entry: A review. Conscious Cogn 2010;19:364-379.

[24] Spence C, Shore DI, Klein RM. Multisensory prior entry. J Exp Psychol Gen 2001;130:799- 832 .

[25] Sullivan MJL, Bischop SR, Pivik J. The Pain Catastrophizing Scale: Development and validation. Psychol Asses;7:524-532.

[26] Titchener EB. Lectures on the elementary psychology of feeling and attention. New York: Macmillan, 1908.

[27] Vancleef LMG, Peters ML. Pain Catastrophizing, but not Injury/Illness Sensitivity or Anxiety Sensitivity, Enhances Attentional Interference by Pain. J Pain 2006;7:23-30.

[28] Van Damme S, Crombez G, Eccleston C. The anticipation of pain modulates spatial attention: evidence for pain-specificity in high-pain catastrophizers. Pain 2004;111:392-399.

[29] Van Damme S, Crombez G, Notebaert L. Attentional bias to threat: a perceptual accuracy approach. Emotion 2008;8:820-827.

[30]Van Damme S, Gallace A, Spence C, Crombez G, Moseley GL. Does the sight of physical threat induce a tactile processing bias? Modality-specific attentional facilitation induced by viewing threatening pictures. Brain Res 2009;1253:100-106.

[31] Van Damme S, Legrain V. How efficient is the orienting of spatial attention to pain? An experimental investigation. Pain 2012;153:1226-1231.

[32] Van Damme S, Legrain V, Vogt J, Crombez G. Keeping pain in mind: a motivational account of attention to pain. Neurosci Biobehav Rev 2010;34:204-13.

[33] Vangronsveld K, Van Damme S, Peters ML, Vlaeyen JWS, Goossens M, Crombez G. An experimental investigation on attentional interference by threatening fixations of the neck in patients with chronic whiplash syndrome. Pain 2007; 127:121-128. 
[34] Van Ryckeghem D, Crombez G, Eccleston C, Liefooghe B, Van Damme S. The interruptive effect of pain in a multitask environment: an experimental investigation. J Pain 2012;13:131-138.

[35] Vlaeyen JW, Linton SJ. Fear-avoidance and its consequences in chronic musculoskeletal pain: a state of the art. Pain 2000; 85: 317-332.

[36] Weinstein S. Intensive and extensive aspects of tactile sensitivity as a function of body part, sex and laterality. In: Kenshalo DR (editor). The skin senses. Thomas, Springfield, 1968. pp 195-200. 


\section{Figure legends}

Fig. 1 - Temporal order judgment data: average of the fitted data for all participants. Data are plotted as a proportion of responses that coincided with the side on which the threatening stimuli were presented (y-axis), as a function of stimulus onset asynchrony (SOA, x-axis), for control trials (broken line) and threat trials (solid line). $\mathrm{R}^{2}=0.99$.

Fig. 2 - Indexes for attentional prioritization of the threatened location (PSS) and for accuracy (JND) (in ms and with standard errors) in control and threat trials $\left(^{*} p<0.05, * * p<\right.$ $0.01)$. 


\section{Table legends}

Table 1 - Singe-subject PSS values (in ms) for control and threat trials. Positive values mean that stimuli on the "neutral" hand had to be presented before stimuli on the hand where pain was expected, to be judged as simultaneous. Negative values mean that stimuli on the hand where pain was expected, had to be presented before stimuli on the "neutral" hand to be judged as simultaneous. 


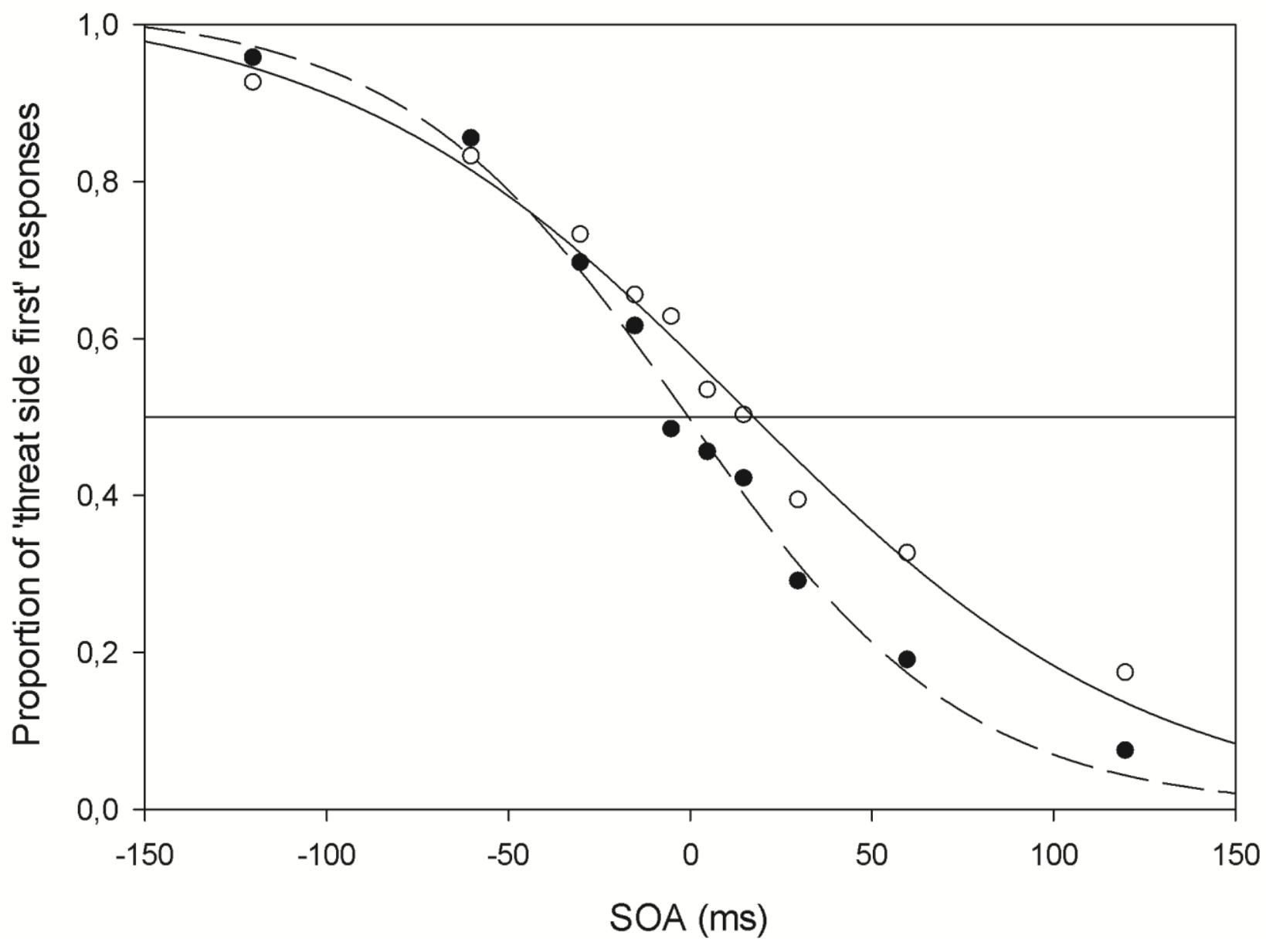




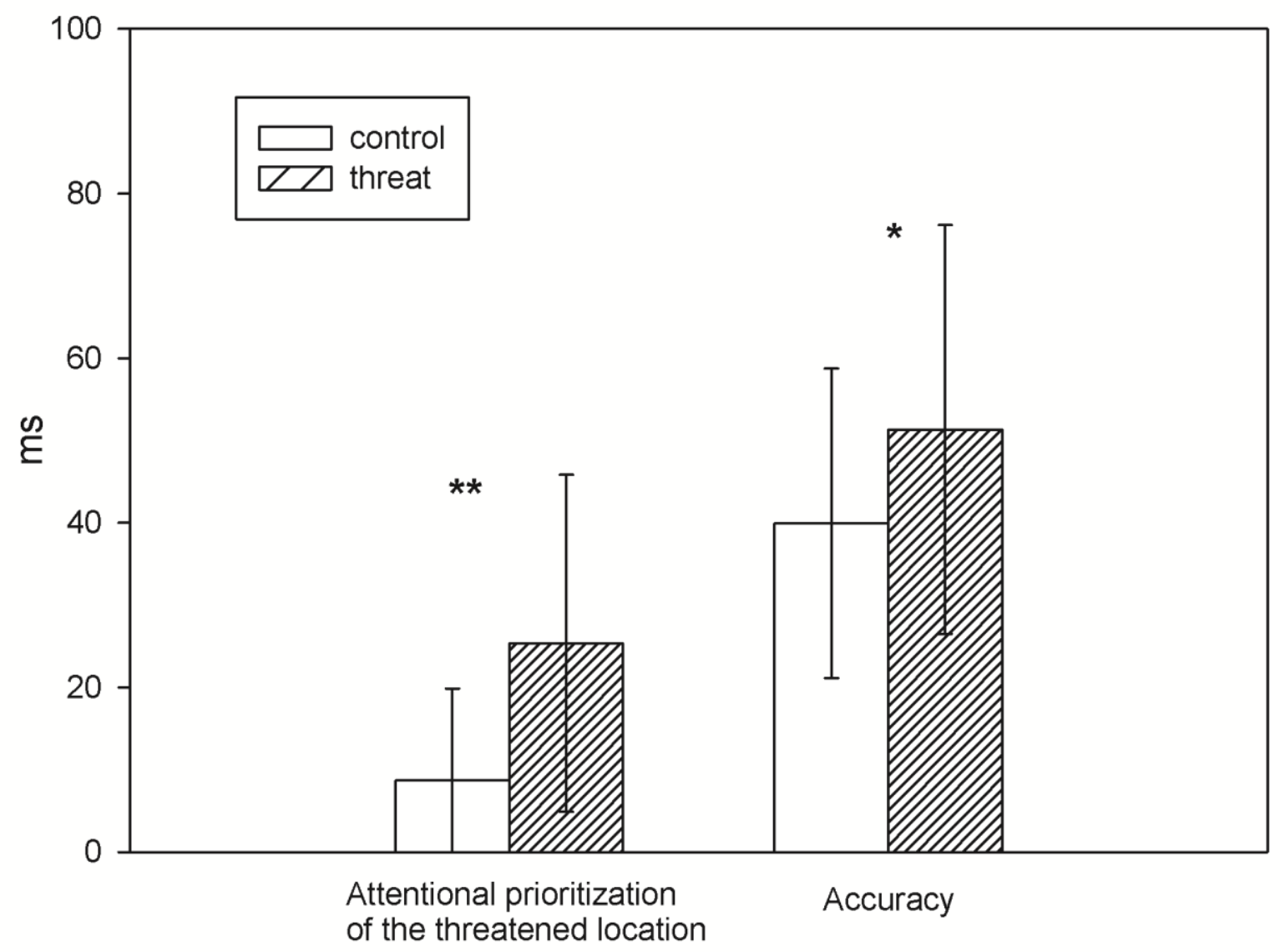




\begin{tabular}{|c|c|c|}
\hline Participant & Control & Threat \\
\hline 1 & 2,93 & 31,50 \\
\hline 2 & 25,40 & 23,30 \\
\hline 3 & 14,16 & 26,06 \\
\hline 4 & 41,47 & 23,96 \\
\hline 5 & $-4,50$ & $-2,83$ \\
\hline 6 & $-7,31$ & 7,08 \\
\hline 7 & 20,11 & 18,10 \\
\hline 8 & 11,58 & 7,94 \\
\hline 9 & 1,25 & 47,14 \\
\hline 10 & 14,67 & 12,19 \\
\hline 11 & $-0,52$ & 67,69 \\
\hline 12 & 8,79 & 24,38 \\
\hline 13 & 5,40 & 30,24 \\
\hline 14 & 0,72 & 4,33 \\
\hline 15 & 5,48 & 33,21 \\
\hline 16 & 1,80 & 74,97 \\
\hline 17 & 9,58 & 39,42 \\
\hline 18 & 2,94 & $-2,30$ \\
\hline 19 & 14,05 & 20,83 \\
\hline 20 & 6,08 & 20,14 \\
\hline
\end{tabular}

